\title{
A Comparative Privacy Research Framework
}

\author{
Philipp K. Masur ${ }^{1}$, Dmitry Epstein ${ }^{2}$, Kelly Quinn ${ }^{3}$, Carsten Wilhelm ${ }^{4}$, \\ Lemi Baruh ${ }^{5}, \&$ Christoph Lutz
}

\begin{abstract}
${ }^{1}$ Department of Communication Science, Vrije Universiteit Amsterdam, Amsterdam, The Netherlands, ${ }^{2}$ Department of Communication and the School of Public Policy, Hebrew University of Jerusalem, Jerusalem, Israel, ${ }^{3}$ Department of Communication, University of Illinois at Chicago, Chicago, USA, ${ }^{4}$ Center for Research on Economies, Societies, Arts and Techniques, Université de Haute Alsace, Mulhouse, France, ${ }^{5}$ Department of Media and Visual Arts, Koç University, Istanbul, Turkey, ${ }^{5}$ Department of Communication and Culture, BI Norwegian Business School, Oslo, Norway
\end{abstract}

\begin{abstract}
The ways in which privacy is defined, perceived, and enacted are contingent on cultural, social, political, economic, and technological structures. Privacy research, however, is often conducted in settings which do not account for variations in how privacy is perceived and enacted. A comparative perspective explicitly addresses this shortcoming by requiring the contextualization of privacy through investigating structural similarities and differences. This paper outlines a comparative privacy research framework, which proposes five interrelated structures (cultural, social, political, economic, and technological) as fruitful units of comparison and disentangles how these structures affect and interact with privacy processes at the micro-, meso-, and macro levels. We conclude by proposing a comparative privacy research agenda, which acknowledges the embeddedness of privacy in such structural settings, and informs efforts to address privacy as a valued outcome through policy formation, education, and research.
\end{abstract}

Keywords: privacy, structures, framework, comparative research, research agenda

\section{This preprint is under review and has not been peer reviewed yet.}

In contemporary societies, privacy plays a pivotal role for enabling individual agency, while both maintaining and altering social structures. For individuals, privacy is critical for the maintenance of the self. The ability to control the flow of information about oneself is fundamental to maintaining autonomy and enabling intimacy, emotional support, and self-validation (Westin 1967). At a societal level, privacy is central to shaping power relations between citizens, government, and commercial actors (Braman 2009). In times of ubiquitous data collection,

Christoph Lutz' work was supported by the Research Council of Norway, Grant \#275347.

Correspondence concerning this article should be addressed to Philipp K. Masur, Department of Communication Science, Vrije Universiteit Amsterdam, De Boelelaan 1081 / 1081 HV Amsterdam, The Netherlands. E-mail: p.k.masur@vu.nl privacy is seen as central to enabling dissent and political expression (Nissenbaum 2010; Solove 2008). Conversely, some scholars question the necessity of privacy for the well-being of democracies (DeBrabander 2020) or even find it to be detrimental to values such as transparency and free speech (Etzioni 1999). In a society where personal information is commodified, privacy-or a lack of thereof-both becomes a critical factor for sustaining or mending social inequality, and at the same time is determined by it (Epstein and Quinn 2020; Papacharissi 2010). The ways in which people perceive and enact privacy differ considerably depending on relevant structural settings (Altman 1977). More specifically, privacy can be regarded as a dynamic process shaped through interaction between individual and collective agency, while exercised within cultural, social, political, economic, and technical structures. 
Yet, despite a large and growing literature, the majority of studies on privacy are mono-national and based on samples from Western, educated, industrialized, rich, and democratic (WEIRD) societies (Henrich, Heine and Nirenzayan 2010; Wu, Vitak and Zimmer 2020). Findings from such studies are often overgeneralized and the role of specific cultural, social, political, economic, and technological differences in shaping privacyrelated outcomes are often neglected or insufficiently operationalized.

A comparative understanding of how such differences influence the ways in which privacy is experienced would inform long-standing debates about why and under what circumstances privacy should be valued, as well as aid the development of universally-applicable empirical approaches (cf. Capurro 2005; Esser and Hanitzsch 2013). Comparative privacy research (CPR) questions the generalizability of findings derived from a single context by inviting scholars to critically assess their own interpretations against structural inconsistencies. By explicitly requiring the identification and description of differences and similarities, CPR forces scholars to question whether theories of privacy developed in a specific culture can adequately explain the phenomenon in other communities, especially those which are marginalized or non-Western. Furthermore, a comparative lens addresses pressing issues such as the applicability of regulatory approaches across regional or national borders, identification of at-risk populations, and the customization of literacy interventions. Findings from CPR can provide practical knowledge about a range of alternatives available to different environments.

But CPR is not simply local methods applied internationally or international networking turned into a scholarly collaboration. Such motivations are insufficient and do not encompass the added value of the comparative approach. While globalization appears to flatten infrastructures, technologies, and services, it also has weakened the heuristic weight of "containers" like nation states, which have diversified internally (Esser and Hanitzsch 2013). A comparative approach to privacy is particularly needed to address the ongoing tension between global infrastructures, platforms, and regulations, and hyper-local, intimate, and personal experiences. Yet, developing a systematic comparative framework for privacy research is challenging due to the breadth and ambiguity of the core concepts. Capurro (2005), for example, warns against comparing concepts of privacy that are derived in different historical or cultural contexts because simple juxtaposition inherently oversimplifies. Comparison for empirical purposes rests on strong assumptions about commonalities that "dissolve into far more complex interrelationships" (Capurro 2005, 45).

With this paper, we aim to avoid these risks and propose a conceptual framework that suggests (a) different structures that can be conceptualized as meaningful units of comparison and (b) roles that such structures might play in shaping privacy dimensions, processes, and outcomes. Finally, we lay out directions for an emerging CPR agenda to address privacy as a valued outcome through policy formation, education, and research trajectories.

\section{Literature Review}

\section{What is Privacy?}

Privacy is an elusive and contested concept (Solove 2008; Nissenbaum 2010). Defining privacy is often the result of an inductive practice that entails examination of situations when individuals or groups think that their privacy has been violated. For example, Warren and Brandeis' (1890) seminal definition of privacy as the "right to be let alone" was a response to the growth of an overly intrusive US press.

Since then, efforts to define privacy both normatively and empirically, fall short in finding a common denominator, settling into either reductive or overly broad conceptualizations (Solove 2008). Nevertheless, approaches to privacy generally fall under two categories (Rössler 2005): intrinsic approaches that see privacy as an end in itself, or functional approaches that try to identify the interrelated values that are protected by privacy, such as intimacy, personhood and autonomy. Conceptualizations of privacy thus often emphasize the protection of the inviolate personality of an individual against intrusions that threaten human dignity. For example, Westin's (1967) conceptualization conceived privacy as a voluntary, temporary withdrawal from others (physically or psychologically), an extraction of the self from social interaction either as in solitude, small-group 
intimacy, or in conditions of anonymity, allowing for autonomy, self-validation, and emotional release.

When conceived as a process of boundary regulation, privacy is perpetually optimized in a dynamic of publicness and privateness, between levels that are realized and desired, through a network of mechanisms that operate as a system (Altman 1975). This view of privacy is inherently social, requiring coordination with others, often in a rule-based process that is managed both individually and collectively (Petronio 2002). More recently, the theory of contextual integrity (Nissenbaum 2010) argues that claims to privacy are claims to an appropriate flow of information in structured social settings with accompanying "canonical activities, roles, relationships, power structures, norms, and internal values" (p. 132). While the theory of contextual integrity has been increasingly applied in empirical research, it has not been systematically transferred into a comparative framework.

As devices and mechanisms for collecting and using personal data have become more ubiquitous, scholarly attention has increasingly focused on information privacy (Lutz et al. 2019). Approaches to privacy in digital environments have focused on interconnectivity, management of relational intimacy, and flow of knowledge about oneself. This line of research has been embellished with ideas such as context collapse (Vitak 2012), imagined audiences (Litt 2012), and an emphasis on the interdependency of privacy behaviors (Baruh and Popescu 2017; Marwick and boyd 2014). Increasingly, scholars call to differentiate between privacy's social (i.e., interpersonal or horizontal), and institutional (i.e., individual-to-institution or vertical) dimensions (Masur 2018; Quinn, Epstein and Moon 2019) and to distinguish among primary, proximal, and distal access and use of data (Bazarova and Masur 2020). The goal of the CPR framework is both to accommodate the use of a variety of privacy conceptualizations and enable systematic ways to scrutinize privacy conceptualizations across structural settings.

\section{Problematizing Privacy Research}

As outlined thus far, conceptualizations of privacy have expanded in their nuance, scope, and reflexivity. They increasingly include refined dimensions related to context, intentionality, metalevel disclosures, collateral information use, and interdependency. Policymakers, too, have signaled privacy's importance and complexity through attempts at regulation, with efforts that include the General Data Protection Regulation (GDPR), Personal Information Protection and Electronic Documents Act (PIPEDA), California Consumer Privacy Act (CCPA), and others. Yet, privacy research (and thus regulation and development of privacy-relevant technology) continues to evolve within epistemological silos (Bräunlich et al. 2020), while privacy theories and conceptualizations continue to be developed and validated through work conducted mostly within WEIRD contexts and among their affluent populations (Wu, Vitak and Zimmer 2020). Privacy research has been criticized as sustaining inherent systemic vulnerabilities by enacting an ethnocentric approach (Arora 2019) and for reifying power structures that are rooted in deterministic, technocentric, capitalistic worldviews that preserve existing forms of marginalization and enable new ones, consisting of the 'privacy rich' and 'privacy poor' (Marwick and boyd 2018).

We believe these discourses and critiques attest to privacy as a vibrant research object, which would particularly benefit from a comparative approach. Such an approach would allow understanding of the ways in which social, cultural, and political differences relate to how privacy is defined, valued, and enacted. Similar to $\mathrm{Wu}$, Vitak and Zimmer (2019), we believe that interrogating the tensions between local and global contexts of design, regulation, and use explicitly addresses some of these fundamental critiques. Thus, through CPR, we strive to consider the broader societal issues connected to privacy in a framework that is sufficiently sensitive to privacy's complex conceptualizations across societies, ideologies and cultures. Before describing the CPR framework, we briefly summarize contextually-situated studies which, despite the lack of a comparative approach, underscore the importance of studying privacy in different social contexts and populations. Then, we provide an overview of a growing number of studies that investigate privacy using comparative approaches. 


\section{Previous Work in Situated Projects}

Contextually-situated privacy studies show how a more nuanced view of privacy has developed to meet the increasing complexity of the field. For example, the study of social media boundary management practices among authoritarian dissidents has revealed the importance of 'slow visibility' privacy strategies to carefully stage the revelation of one's political beliefs and activities (Pearce, Vitak, Barta 2018). Examination of privacy practices of teens has provided a more holistic view of privacy regulation practices, one that acknowledges the privacy management dynamic between individuals and networks (Marwick and boyd 2014). Other research has identified the contribution of factors such as privacy fatigue (Marwick and Hargittai 2019) to networked privacy strategies (De Wolf 2020).

Studies of marginalized populations have revealed the embeddedness of Western ideals for privacy, disabusing conceptions of privacy seekers as monolithic. For example, work conducted among Australian Aboriginal communities, in which the social is rooted in relatedness, exposes technological bias for a privacy that is premised on individual access and control (Rennie, Yunkaporta and Holcombe-James 2018). The communities' reliance on mobile devices for Internet connectivity coupled with norms of device sharing contrast with the individualistic privacy management practices embedded in mainstream platforms and devices, exposing community members to "specific privacyrelated ordeals, including identity violations and unauthorized access to financial accounts" (p. 1305). Likewise, examination of the reconciliation process between the Canadian government and its Indigenous populations demonstrate how liberal approaches to privacy reproduce structures of colonization when privacy is used as justification to erase documents demonstrating the extent of statesanctioned violence against Indigenous communities (Fullenwieder and Molnar 2018).

Finally, in authoritarian societies, uncertainty about technological and sociopolitical sanctions by governments counter online dissent or collective action (Sanovich, Stukal and Tucker 2018). In such contexts, the 'cost' of political expression may be different than the 'risk' of sanction, exposing these as inequivalent concepts (Dal and Nisbet 2020).
This confronts the interchangeability of these two ideas when exploring important privacy mechanisms such as the privacy calculus (e.g., Dienlin and Metzger 2016). In sum, these contextually-situated works illustrate how cultural, technological, political, economic, and social settings influence how privacy is perceived and enacted. We argue that a comparative approach challenges simplistic conceptual and empirical transferability, thereby deepening the understanding of privacy.

\section{Previous Comparative Privacy Research}

While its volume is limited, CPR comprises an active trajectory within privacy studies that has highlighted important structures along which privacy can be evaluated. Often studies rely on national boundaries to offer descriptive differences in privacy decision-making and behaviors (e.g., European Commission 2019; Trepte and Masur 2016). Political geography offers a mechanism for understanding difference, though it is sometimes, somewhat imprecisely, interpreted as offering a cultural lens (Livingstone 2013). Other, more culturally-aligned studies call attention to underlying dimensions and values (e.g., Hofstede's [1984] notions of individualism and collectivism) as mechanisms to explain distinctions (e.g., Li, Rho and Kobsa 2020; Miltgen and Peyrat-Guillard 2014). These works generally hinge on the premise that nations have solidified a distinctive national culture, perpetuated through institutions such as family, education, media, and law. Such studies would benefit from approaches that investigate sources and consequences of variation within national culture.

Some comparative studies have emphasized the political contexts of privacy by uncovering difference and similarity attributable to political systems, ideologies or regulatory regimes (Bignami 2007; Koops et al. 2017). Comparing across political contexts highlights the significance of privacy to democratic processes, such as voting (Bennett 2013), yet also that responsibility for privacy shifts between the individual and state, depending on the political system or regulatory regime (Pearce, Vitak and Barta 2018).

The role that different forms of privilege-e.g. income, education, youth, and race-play in the 
attainment of privacy outcomes has been particularly revealing of societal inequalities, offering pointed critique to utopian expectations for the digital realm. One rich area of study is privacy literacy, which focuses on the knowledge and skills needed to accomplish privacy online (Park 2013; Masur 2020). Such comparative studies have demonstrated that traditional markers of disparity, such as gender and income, are also markers of privacy literacy, and that privacy literacy is directly linked to privacy outcomes (Epstein and Quinn 2020; Kezer et al. 2016).

Finally, comparative research has drawn attention not only to the ways in which individuals experience privacy in various technological contexts, but also to the affordances that various technologies offer for privacy enactment. Studies in this area have highlighted similarities and differences in privacy threats and protections across different online spaces (Pekárek and Pötzsch 2009), and also that technological architectures influence behavioral norms that relate to privacy (Papacharissi 2009; Lee and Yuan 2020). Such work is critical to reinforcing privacy by design efforts (Tamò-Larrieux, 2018), as well as for developing appropriate policies and regulatory frameworks for privacy protection (Wu, Vitak and Zimmer 2020).

In sum, comparative studies have focused on important intersections between privacy and culture, structural inequities, and political ideology and likewise have emphasized the economic and socio-technical dimensions of privacy behavior and decision-making. A framework that offers a systematic way of thinking through the values, challenges, and methodological considerations of CPR is, in our view, the necessary next step in developing this trajectory of study.

\section{Comparative Privacy Research Framework}

The goal of this paper is to develop a framework for systematic CPR, building on existing work while addressing its limitations. First, we describe five interrelated "structures" across which privacyrelated variables or processes can be meaningfully compared. We acknowledge that any attempt at classifying societal settings is inherently limited

\footnotetext{
${ }^{1}$ Note that we interpreted these theories rather loosely to arrive at useful units of comparison.
}

and oversimplifying. Nonetheless, as the dispersed threads of literature summarized above suggest, differentiating cultural, social, political, economic, and technological structures provides a fruitful framework for explaining similarities, differences, or inconsistencies in privacy-related outcomes. Second, we disentangle and systematize the ways in which these structures influence or determine privacy outcomes and processes. Furthermore, we acknowledge the duality of these structures, as both influencing and, at the same time, reifying the collective practices of groups and individuals. Third, we acknowledge that these structures may affect privacy outcomes on the micro-, meso-, and macro-levels by emphasizing that a comparative perspective is not limited to aggregated, macrolevel privacy variables, but also highlights differences in meso- or micro-level processes (e.g., behaviors on different platforms).

\section{Conceptual Framework}

\section{Structural Units for Comparison}

Comparative research generally involves contrasting a minimum of two macro-level units (e.g. countries or social milieus), during at least one point in time, with at least one object of investigation (Esser and Hanitzsch 2013). A systematic literature review of 147 articles that classify as comparative communication research (So 2018) shows that most (56\%) focus on countries or nations as structural units. However, 8.7\% also compare certain systems (mostly political systems), $6.5 \%$ specifically conduct comparisons across cultures, $1.6 \%$ compare different "people" (e.g., based on sociodemographics), and $1.1 \%$ compare different markets (economic structures). As a means of methodological bracketing (Phipps 2001) that builds on these findings, we propose five central units for comparison: cultural, social, political, economic, and technological structures (see Table 1). On the one hand, these five structures are inspired by sociological theories that differentiate social systems (Luhmann 1995) or social fields (Bourdieu 1993) as distinct macro-structures of society. ${ }^{1}$ On the other hand, we summarized the 
Table 1. Overview of Structures

\begin{tabular}{lll}
\hline Structures & Description & Examples \\
\hline Cultural & $\begin{array}{l}\text { Collectives of people with shared } \\
\text { identities, values, or practices }\end{array}$ & $\begin{array}{l}\text { Collectivist vs. individualist cultures; } \\
\text { specific subculture compared in different } \\
\text { geographic locations }\end{array}$ \\
Social & $\begin{array}{l}\text { Sociological concepts that classify } \\
\text { people into groups of similar } \\
\text { characteristics }\end{array}$ & $\begin{array}{l}\text { Adolescents vs. elderly; } \\
\text { males vs. females; clustering based on soci } \\
\text { Political }\end{array}$ \\
$\begin{array}{l}\text { Political concepts that differentiate } \\
\text { political and/or regulatory systems }\end{array}$ & $\begin{array}{l}\text { Democracies vs. autocracies; } \\
\text { levels of surveillance; voting systems }\end{array}$ \\
Economic & $\begin{array}{l}\text { Economic concepts that distinguish } \\
\text { markets, commons, or economic } \\
\text { power structures }\end{array}$ & $\begin{array}{l}\text { Market economy vs. planned economy; } \\
\text { monopolistic vs. oligopolistic market } \\
\text { systems }\end{array}$ \\
\hline Technological & $\begin{array}{l}\text { Types or families of technological } \\
\text { environments }\end{array}$ & $\begin{array}{l}\text { Ephemeral vs. persistent communication; } \\
\text { comparison based on affordances }\end{array}$ \\
\hline
\end{tabular}

extant comparative privacy research to identify these structures (see the work discussed in the previous chapter and the review by So 2018). They thereby reflect the often expressed need to conduct not only cross-cultural or cross-national comparisons, but also echo calls for cross-political as well as cross-platform investigations (e.g., Matassi \& Boczkowski 2021).

Moreover, the structures and their boundaries emerged iteratively since 2018 through intense deliberations among the author team, who itself draws on diverse methodological, theoretical and epistemological backgrounds. Several conferences and workshops organized by the author team and dedicated to the topic of comparative privacy research further shaped and sharpened the framework and its structural units for comparison (see https://comparativeprivacy.org/ for an overview). These events also engaged the wider privacy research community to expose the framework and the five structures to useful feedback. We recognize that these structures are not exhaustive and other units of comparisons may emerge if scholars with different disciplinary backgrounds or foci engage with privacy. Despite this limitation, we view them as dynamic and interrelated, simultaneously shaping human behavior and being shaped by the exercise of agency. In the following subsections, we describe each structure in more detail.

Cultural structures. Historically, much comparative research has focused on cross-cultural comparisons and macro-level units such as countries (So 2018), which are often conflated with cultural differences (Taras, Steel and Kirkman 2016). Although a narrow focus on such units has been criticized (Livingstone 2013), differentiating people based on their cultural affiliation can nonetheless be meaningful. From an anthropological perspective, one can argue that people identify or are identified with certain cultures (e.g. national cultures, age-groups, gender), which in turn shape both how they are perceived by others and also how they enact their own identity. Culture thereby can be understood as a collective of individuals that shares values and practices (Jahoda 2012; Kroeber and Kluckhohn 1952), and as a primary framework for agency (Goffman 1974).

The influence of cultural structures on the enactment of privacy is well noted in the literature (e.g., Miltgen and Peyrat-Guillard 2014; Trepte et al. 2017), and it allows for the investigation of difference and similarity on all levels of society. For example, Murphy's (1984) anthropological study of the use of veils among men from Saharian 
Tuareg culture suggests that veils aid the management of social distancing because they hide relevant social cues, allowing men to be socially engaged while remaining aloof. In Western cultures, research on interpersonal relationships similarly suggests that withholding sensitive information or concealing affective states may support relationship initiation by adding ambiguity (e.g., Norton, Frost and Ariely 2007). A comparative approach would contribute to understanding how distance and ambiguity are utilized in different cultures to accomplish privacy.

Cultural structures, however, are not limited to groups with shared ideas, customs, and social behaviors within national boundaries, as they can also refer to subcultures or to any group who identify themselves as a collective (e.g., Amish people in the US, hip hop culture, quantified-self movement). By requiring specific articulation, the CPR framework proposes to systematically address the challenge posed by nested, overlapping, and concurrent cultural influences.

Social structures. Regardless of multiple cultural identities, people are also sorted into social clusters (by socioeconomic status, educational levels, etc.), which may constrain, enable, or reify ways in which privacy is perceived and enacted. From a sociological perspective one can compare structural arrangements (e.g., institutions or classes), as well as practices, roles, and social norms, using both quantitative and qualitative approaches (Lutz 2016). As questions about privacy are often interwoven with questions about social inequality and power, CPR addresses how, on one hand, characteristics of social structures relate to differences in privacy access and, on the other hand, how contexts of privacy may act as a marker of status.

Alternatively, socio-psychological approaches might reveal how socially shared perceptions of privacy, and the ways in which it is balanced with other needs (e.g., personal safety), are linked to different life stages (Peter and Valkenburg 2011). Such perspectives are valuable because they expose the social construction of privacy at all levels of society, as well as in all stages of individuals' development.

With privacy increasingly a commodity (Papacharissi 2010), further analyses of social structures also offer a view of privacy at the social margins (Marwick and boyd 2018). Social structures are important units of comparison because they emphasize human behavior as relational. However, it is important to recognize that cultural and social structures are not always distinct. For example, an individual may identify with a certain gender, and therefore feel part of a certain culture (e.g., LGBTQ+), and in turn be influenced by shared values and norms within that group. However, the same individual may at times belong to a social cluster (e.g., by sex), which may constrain their ability to enact or control their desired level of privacy. As such, differentiating cultural (e.g., gender) from social categories (e.g., sex) is often a useful heuristic, despite difficulties attributable to their intersections.

Political structures. Privacy is inherently political and increasingly regulated. Societies exist in differing political systems and regulatory regimes (e.g., democracy vs. autocracy, PIPEDA vs. GDPR), which may determine the conditions under which individuals, groups, or organizations experience the flow of personal information. The ways in which privacy and data protection are regulated and implemented are also often contingent on political systems. Political structures therefore refer to governmental practices such as surveillance (Lyon 2014) or limits to free speech, and involve their impact on, for example, individual handling of private information or perceptions of privacy. Comparison of directives, acts, and other legal texts across diverse political systems reveals distinctions in privacy's conceptualization $(\mathrm{Wu}$, Vitak and Zimmer 2011) and in notions about who can be entrusted with its protection. For example, data localization is seen as a way of countering data colonialism and leveling the playing field for the Global South, which rely on services and infrastructures owned by Western conglomerates (Belli 2021). On the other hand, data localization is criticized as enabling domestic surveillance by authoritarian regimes (Belli 2021; Taylor 2020). CPR can contrast how different regulatory contexts emphasize 'freedom from' intrusions over 'freedom to' attain self-actualization (e.g., Koops et al. 2017; Masur 2020).

Economic structures. Differentiation between economic structures (e.g. monopolistic vs. competitive, open vs. closed markets) also provides valuable insight into privacy. Specific economic 
environments place different premiums on private information and provide alternative incentives for protecting, invading, and exploiting individual privacy. Market structures may thereby shape interactions between individuals who use certain services, companies which provide those services, and regulators who aim to align information flows with data protection law (Bazarova and Masur 2020). Data brokers and the provision of financial incentives for privacy-compromising internet use (e.g., Facebook Zero) have accentuated privacy's economic underpinnings. The exploitative practices of companies such as Alphabet/Google and Facebook, and their business models of data accumulation (Zuboff 2019), are rarely systematically compared for privacy implications outside of security research (e.g., Daryabar et al. 2016). CPR on varying economic structures can therefore highlight privacy's significance to the ways in which inequality and power are distributed and negotiated.

Technological structures. Individuals increasingly communicate and conduct their daily lives in technological environments, which depend on data-based business models and afford different conceptions and mechanisms for privacy (e.g. fintech, IoT). Communication scholars have long studied how media platforms (e.g., social network sites, online shopping websites) and technologies (e.g., smartphones, fitness trackers) restrict and enable information flows and privacy. Matassi and Boczkowski (2021), for example, show the value of cross-media, cross-platform and cross-national comparisons. In everyday practice, most users combine social and other media across multiple platforms. Comparative perspectives in combination with particular structures "foster a denaturalization process whereby a case once thought to be unique is reconceptualized in light of other, similar or different, cases" (p. 208).

CPR allows for the analysis of similar families of technological arrangements (i.e., those which provide similar affordances), in contrast to specific devices or platforms which are prone to ephemerality. In other words, it is arguably more fruitful to compare affordances (Evans et al. 2017) of technological structures (e.g., high vs. low editability, persistent vs. ephemeral communication) and their influences on privacy outcomes. A focus on technological structures would also include a systematic comparison of communication modalities (e.g., face-to-face vs. mediated, online vs. offline) for how privacy norms are negotiated, boundaries managed, and intimacies experienced (e.g., Croes and Antheunis 2021).

\section{The Role of Structures in Shaping Privacy}

We envision these five structures as independent variables in the CPR framework. A first determination should thus be made as to whether the structural arrangement (a) directly influences a privacy-related outcome or (b) moderates a privacy-related process. Figure 1 provides an illustration of both types of influences. In its most basic form (Fig. 1A), CPR involves the comparison of at least two structural units (e.g., two or more political systems, two or more online platforms) with respect to at least one object of investigation (e.g., privacy concerns, selfdisclosure). Objects of investigation must be functionally equivalent (Esser and Hanitzsch 2013; Maurice 1989). For example, if one social media platform offers elaborate privacy settings and another does not, a meaningful comparison of how people use privacy settings on these platforms may be impossible, though the presence or absence of a structural element can offer a meaningful comparison (e.g., whether or not countries have explicit privacy regulations).

Identified structures can also influence privacyrelated outcomes indirectly. In many cases, structures can be investigated as inhibitors or facilitators of privacy-related processes (Fig. 1B), as they may also influence the (causal) relationship between two privacy-related variables. For example, the relationship between online privacy concerns and online information disclosure may depend on technological structures. Thus, a permanent status update on Facebook, which is persistent but can have different levels of audience control, can be contrasted with posting a story on Instagram, which has fewer audience controls, but is inherently ephemeral.

The interaction of structural arrangements to influence privacy-related outcomes also may be significant. Structures should not be regarded as ontologically distinct; they may overlap, interact, or be nested within each other. Figure 2 provides illustration of potential interactions between 
A
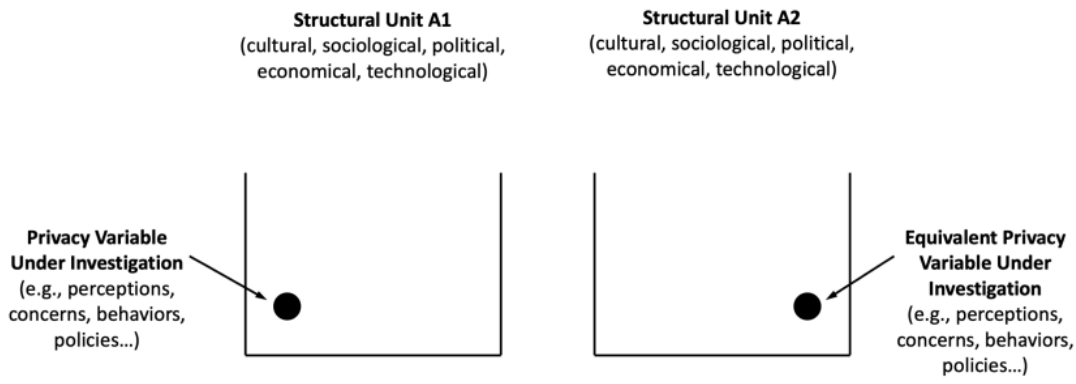

B

Structural Unit A1

(cultural, sociological, politica economical, technological)

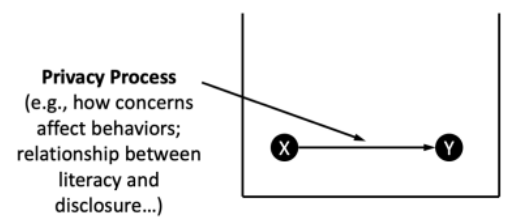

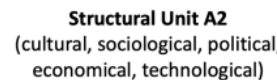

economical, technological)

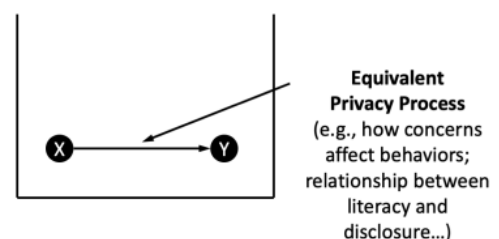

Figure 1. How structural units shape privacy (cf. Esser and Hanitzsch 2013). A: Direct influence on privacy-related variables. B: Moderating influence on privacy-related processes.

structural arrangements. First, it is possible for one structural setting to be nested within a larger structural setting. From a theoretical perspective, this has important implications as subunits within one superstructural unit may share more similarities than differences when compared to subunits within another superstructural unit (e.g., despite heterogeneity within the Israeli or Chinese start-up subculture, each set of subcultures may share similarities due to being located in their respective countries; Fig. 2A). Specificity is a powerful heuristic for determining the influences of each type of structure, hence its importance at all levels.

Second, non-nested structural settings may simultaneously influence privacy-related outcomes (Figure 2B). For example, in studies in which several privacy-related variables (e.g., privacy literacy or data protection behaviors) are measured using a survey that has been translated and administered in several locations, privacy outcomes can be compared across cultures, but potentially also across political systems. Structural units (A and B) are hence non-nested. Such research designs are particularly interesting as they allow researchers to investigate potential interactions between two structural units, culture and political system. A fruitful investigation may examine how cultural factors, such as individualism or power-distance, interact with political regimes in predicting public support for data localization laws. It is important to note that both examples of structural configurations (Fig. 2A and 2B) may oversimplify the complex entanglement of two or more structural units, which are often not theoretically distinct or have considerable overlap. Specification of the structural units again helps to interrogate and describe such entanglements. 


\section{A}

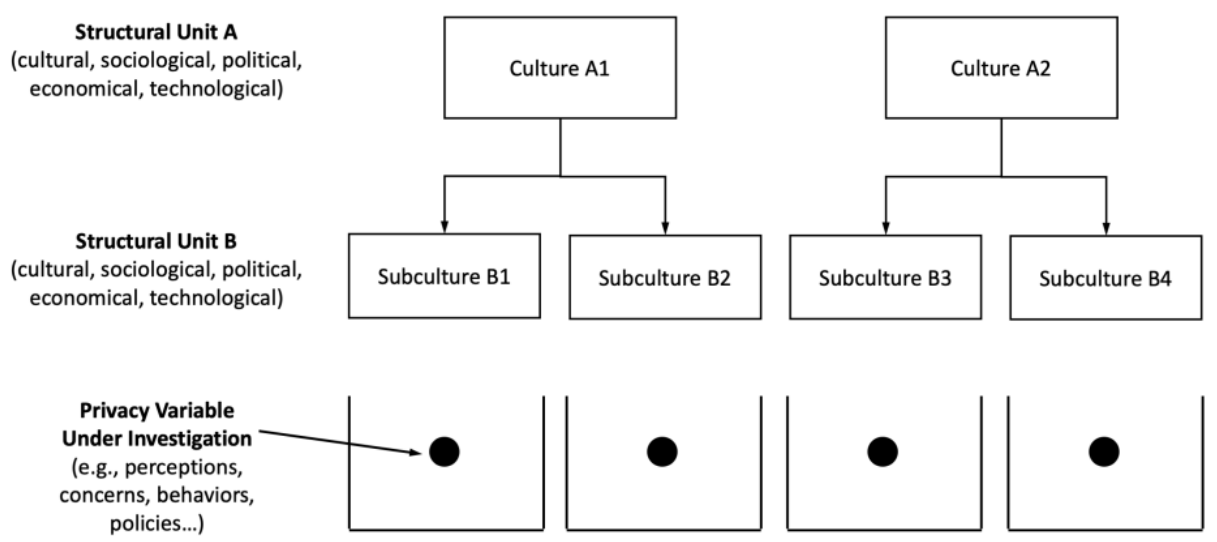

B

Structural Unit A (cultural, sociological, political, economical, technological) Structural Unit B
(cultural, sociological, political,
economical, technological)

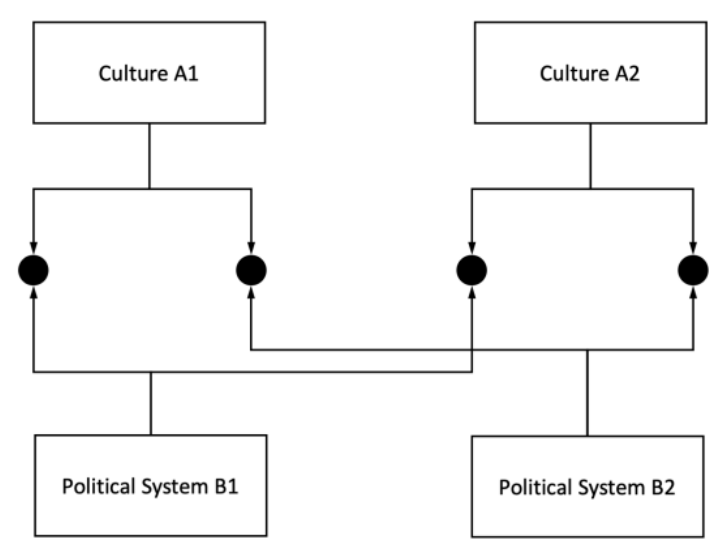

Figure 2. Potential interactions between structural arrangements. A: Nested structures. B: Cross-classified structures.

\section{Level of Analysis and Level of Aggregation}

We turn next to identifying (a) the level of analysis on which structural configurations are situated and (b) the level of analysis on which they influence or moderate privacy-related outcomes. First, the traditional macro-, meso-, and micro-level differentiation (Turner 2005) is useful for articulating the level on which a structural unit of comparison is located. While these three levels are inherently interdependent, systematic analysis requires distinguishing between them as they dictate units of observation, type of aggregation, and analytical procedure. We anticipate that CPR will frequently focus on macro-level units (e.g., cultures, nations, political systems), but comparisons on a meso-level (e.g., different organizations, or technological environments) or micro-level (e.g., social contexts or situations) may be equally important and fruitful. Despite its higher analytical complexity, combining several structural units or levels of analysis, is a promising avenue for future research. For example, exploring cultural (macro-level) and technological structures (mesolevel) would allow examination of international communication of families concurrently using WhatsApp and WeChat (Matassi and Boczkowski 2021)

Second, objects of investigations are embedded in structural units of comparison, so they must be on the same level or lower; higher level structures may moderate privacy-related processes on the same, and also lower, levels. For example, national cultures (macro-level) might affect how individuals perceive certain risks (micro-level), and in turn 
their data protection behavior (Trepte et al. 2017). Similarly, meaningful comparisons of data protection legislation (macro-level) across different nations (macro-level) or public documents can be made (Bosler and Wilhelm 2017). Importantly, comparison of privacy-related variables across specific structural units will always be aggregated to the level of the structural units. For example, to understand how users of different social network sites vary in their privacy protection strategies, privacy behavior is measured on the micro-level (e.g., for each individual), but then aggregated to the higher level for the actual comparison (e.g., Facebook vs. Twitter users).

\section{Privacy as an Object of Comparative Research}

Given the breadth of conceptual work on privacy (Acquisti, Brandimarte and Loewenstein 2015; Masur 2018), researchers must make a conscious decision about which aspects, processes or dimensions of privacy to investigate, as a function of their goals and epistemology. In many instances, comparative research may focus on comparing individuals and their perceptions, attitudes, and behaviors across structures. However, it is equally important to investigate contextual or societal dimensions of privacy. In the following, we discuss each of these perspectives in more detail, linking them to existing theoretical approaches of conceptualizing privacy.

Individual (micro) level. Much research on privacy is based on socio-psychological theories explaining privacy-related personal characteristics, attitudes, and privacy behaviors. Such a perspective helps to conceptualize and identify micro-level aspects of privacy for the purpose of CPR. Individualistic theories of privacy (cf. Bazarova and Masur 2020), for example, emphasize individuals' control of personal information in social interactions by focusing on perceptions of privacy (e.g., desired vs realized level of privacy; Altman 1975), the ways in which individuals can (or cannot) regulate their privacy in various situations (e.g., Masur 2018), and an individual's amount of information control (Crowley 2017) or self-disclosure (Omarzu 2000). These individualistic approaches may incorporate additional variables such as privacy cynicism (Lutz et al. 2020), privacy literacy (Masur 2020), or privacy heuristics (Sundar et al. 2013) to investigate privacy processes in specific structural settings. The CPR framework encourages testing the generalizability of such approaches across diverse structural settings.

Typological or dimensional approaches distinguish specific aspects of privacy (e.g., Westin 1967, Burgoon 1982), and these can be compared across structures. For example, examination of privacy states (e.g., solitude, anonymity) and their specific functions (e.g., autonomy, protected communication; cf. Westin 1967) across varying political structures demonstrates that while anonymity may function as a form of autonomy in a democratic society, it is used as protection against both state and ideological opponents under autocratic regimes (Pearce, Vitak and Barta 2018). Similarly, the willingness to disclose financial information is also contingent on the ways in which governments handle such information (Eurobarometer 2011).

Finally, actor-based conceptualizations of privacy are useful to disentangle specific perceptions, concerns, attitudes, or behaviors of individuals with regard to particular actors. Comparing perceptions, attitudes, and concerns across privacy's horizontal and vertical (which may further be subdivided into governmental and commercial) dimensions against varying political, economic, or technological structures may yield important insights into privacy concern at the individual level, potentially introducing more finegrained remedies. For example, while social media users may be primarily concerned about horizontal privacy relationships (Quinn, Epstein and Moon 2019), elites, such as policymakers, engage in vertically-oriented privacy critique (Epstein et al. 2014). CPR can highlight such disjunctures and inform future educational and policy efforts.

Contextual (meso) level. A caveat of microlevel approaches is that they conceive individuals as monadic elements and ignore the relational aspects of communication and privacy processes. Explicit focus on relational aspects of privacy practice, among institutions and organizations, represent meso-level aggregation. Contextual approaches to privacy (Nissenbaum 2010) emphasize that privacy perceptions and behavioral responses, and thus privacy violation experiences, are related to contextual factors. For example, 
referring to the privacy paradox, behaviors tend to be more consistent with privacy concerns in commercial interactions, but less so on social media (Lutz and Strathoff 2014). Specific expectations for information flow may differ considerably across age groups, countries, or as a result of the availability of privacy management options on different platforms. CPR encourages both deepening and expanding systematic analysis of such contextual factors.

Networked approaches to privacy focus on the interconnectedness of individuals in managing and handling their privacy (Marwick and boyd 2014; Petronio 2002), describing group processes such as boundary management and decision-making based on shared norms and rules. Yet, the notion of group privacy presents vexing questions such as whether groups can claim privacy harms or how group privacy interests can be balanced with the individual's interest in informational selfdetermination (Taylor, Floridi and van der Sloot 2017). CPR offers a mechanism for systematic investigation of the role of structural factors in meso-level processes that impact key privacy outcomes.

Societal (macro) level. Comparison across structural units (e.g., nations or political systems) will result in the aggregation of individual and contextual data at the macro-level. Such comparisons are valuable in their own right, given the inherent tension between global information infrastructures and localized user experiences. Identifying explicitly macro-level dimensions of privacy, instead of relying on micro-level data generation, is equally important $(\mathrm{Wu}, \mathrm{Vitak}$ and Zimmer 2020). The analysis of privacy regimes (e.g., national data protection legislation, multinational harmonization efforts) is also an important object of study in privacy research (e.g. Park,2008). Legal frameworks that dictate requirements for data localization (Selby 2017) and data protection officers (Medzini 2021), or lay out approaches to regulation of social media platforms (Stockmann 2020), are important factors in shaping privacy norms and attitudes at national, regional, and global levels. For example, the GDPR-a regional legislative effort-has had a global, yet arguably differential, impact on privacy practices worldwide (Li, Rho and Kobsa 2019). CPR enables unpacking the dynamics between such macro-level processes.

\section{The Road Ahead: Discussion, Research Agenda, and Outlook}

CPR emphasizes the embeddedness of privacy and related concepts in structural settings. The proposed framework outlines five interrelated structures as potentially meaningful units for comparative analysis and further describes their individual and combined effects on the individual, contextual, and societal levels. We believe the proposed framework can advance the scope and nuance attributable to privacy and its outcomes. To focus on several fundamental questions facing privacy researchers, and inform privacy regulation and technology design, we outline the following important and sustainable trajectories for CPR moving forward.

First, we critique the ethnocentric nature of past privacy conceptualization efforts. A comparative approach inherently challenges uniform approaches to privacy and holds the promise of adding nuance by exposing Western assumptions that are irrelevant to different cultural, political, economic, or technological settings. Moreover, such an approach confronts the view of privacy as an ideal state, and considers the instrumental value that a lack of privacy holds in macro- or meso- contexts. The CPR framework is useful for both testing established theoretical constructs and developing grounded privacy theory in new structural contexts.

Second, privacy issues are specifically linked to both global and local power structures. Multinational companies and their practices contrast with local data protection regulations and individual-level needs and preferences. Bridging the global and the local is, and will be, pivotal for understanding the continually changing and increasingly complex relationships between individuals, commercial players, and governments. The CPR framework can be used to examine use practices of global platforms across cultural contexts, to unveil the values and assumptions embedded in technological solutions, or to understand unintended effects of local policy resolutions. 
Third, CPR is critical to understanding how privacy is perceived and enacted at the social margins. An explicit focus on structural factors that determine specific privacy needs, goals, and constraints of those who are marginalized will help to design respective and respectful solutions, technologies, and practices. When comparing countries, platforms, or social clusters, it is imperative that privacy research moves from studying primarily WEIRD societies, and also from treating privacy as uniform within societies. The CPR framework can be an important tool to understanding the role of privacy in reducing or enhancing social inequality.

\section{Limitations and Future Perspectives}

First, by the very nature of placing "methodological brackets" (Phipps 2001) around complex and interrelated ideas, this proposal is a compromise between different points of views and opinions. While working on this framework, our international team of authors had in-depth discussions about terminology (e.g., about terms such as "structures" or "cultures"), classification (e.g., which types of structures to include), theoretical perspectives (e.g., what theories to review for a comparative lens), and applications (e.g., use of the framework across methodologies). It is important to acknowledge these tensions when viewing the proposed framework as the final product presented in this paper. As such, we view this framework as an initial step in thinking about CPR, one which should be used with caution and subjected to critical empirical investigation. For example, we explicitly focused on five macro-level structures, as we deem these particularly important for a nuanced understanding of privacy phenomena, although other macro level structures might be worth considering and can broaden the spectrum. Yet, the outlined CPR framework may also work with individual-level "structures" such as psychological or between-person differences (e.g., personality characteristics) or within-person processes (e.g., comparing different situations). We thus invite scholars with different backgrounds to explore the benefits of adopting a comparative approach for their particular mode of investigation.

Second, practical or financial reasons may prevent researchers from studying several structures simultaneously. Any such bracketing of structural factors represents a compromise and is inherently limiting. The utility of this framework thus lies in offering ways to make informed bracketing decisions when conducting comparative work. We underscore that these particular structural arrangements are intertwined and need to be considered in relation to each other.

Third, our framework does not explicitly address temporal dynamics in both structures and outcomes. An extension of our framework might add a longitudinal, or cross-temporal, component (Esser and Hanitzsch 2013), as local and global events may change perceptions, practices, and markets. As illustration, during the writing of this paper, the authors experienced the COVID-19 pandemic in different countries and under various structural settings (e.g., living in family groups or only with a partner, working in an office or at home). In doing so, we recognized that privacy research is affected by global events, which create new areas of study-such as privacy concerns related to contact-tracing apps-and change how structural arrangements might play into individuallevel privacy perceptions, such as increasing acceptance of governmental monitoring of private life. It is our hope that this framework will help to clarify and surface these particularly pressing conditions.

Finally, the proposed framework requires empirical application and testing. Empirical CPR research thereby should build on established comparative methodologies (Esser and Hanitzsch 2013). For example, content analysis could be used to compare the media coverage about privacy across different countries or media markets. Comparative survey studies, likewise, can tap into similarities and differences across various structural settings (Li, Rho and Kobsa 2020; Miltgen and Peyrat-Guillard 2014). Methodological rigor is required to appropriately model the structural influences outlined in the CPR framework. Future research should systematically identify and analyze the methodological challenges and pitfalls of using this framework.

\section{Conclusion}

With this paper, we proposed a CPR framework in which we aim to systematize attempts 
to understand and study the embeddedness of privacy in diverse structural settings. We introduced five interrelated structures (cultural, social, political, economic, and technological) as useful starting points for comparison. We further described how these structures affect privacy- related outcomes on micro-, meso-, and macrolevels. We hope that this framework provides a basis for future, more systematic work and thereby contributes to global, as well as local, efforts to address pressing privacy issues.

\section{References}

Acquisti, A., L. Brandimarte and G. Loewenstein. 2015. Privacy and human behavior in the age of information. Science 347 (6221):509514.

Altman, I. 1975. The Environment and Social Behavior: Privacy, Personal Space, Territory, Crowding. Pacific Grove, CA: Brooks/Cole Publishing.

Altman, I. 1977. Privacy regulation: Culturally universal or culturally specific. Journal of Social Issues 33 (3): 67-83.

Arora, P. 2019. Decolonizing privacy studies. Television \& New Media 20 (4):366-378.

Baruh, L., and M. Popescu. 2017. Big data analytics and the limits of privacy self-management. New Media \& Society 19 (4): 579-596.

Bazarova, N. N. and P. K. Masur. 2020. Towards an integration of individualistic, networked, and institutional approaches to online disclosure and privacy in a networked ecology. Current Opinion in Psychology $36: 118-123$.

Belli, L. 2021. CyberBRICS: Cybersecurity Regulations in the BRICS Countries. Berlin: Springer.

Bennett, C. 2013. The politics of privacy and the privacy of politics: Parties, elections and voter surveillance in Western democracies. First Monday 18 (8).

Bignami, F. 2007. Privacy and law enforcement in the European Union: The Data Retention Directive. Chicago Journal of International Law 8 (1):233-255.

Bosler, S. and C. Wilhelm. 2017. La politique des études d'usage: Une méta-analyse internationale des études sur les médias numériques. Les Enjeux de l'Information et de La Communication 18(3a).

Bourdieu, P. 1993. The field of cultural production. In The Field of Cultural Production: Essays on Art and Literature, ed. R. Johnson R, 29144. New York: Columbia University Press.

Braman, S. 2009. Change of State: Information, Policy, and Power. Cambridge, MA: MIT Press.

Bräunlich, K., T. Dienlin, J. Eichenhofer et al. 2020. Linking loose ends: An interdisciplinary privacy and communication model. New Media \& Society. Epub ahead of print 12 Feb 2020.

Burgoon, J. K. .1982. Privacy and communication. Communication Yearbook 6:206-249.

Capurro, R. 2005. Privacy. An intercultural perspective. Ethics and Information Technology 7 (1):37-47.

Chen, S. and M. A. Williams. 2009. Privacy in social networks: A comparative study. PACIS 2009 Proceedings. https://aisel.aisnet.org/pacis2009/81

Croes, E.A.J. and M. L. Antheunis. 2021. Perceived intimacy differences of daily online and offline interactions in people's social network. Societies 11 (1):13.

Cohen, J. E. 2013. What privacy is for. Harvard Law Review 126 (7): 1904-1933.

Crowley, J. L. 2017. A framework of relational information control: A review and extension of information control research in interpersonal contexts. Communication Theory 27 (2):202-222.

DeBrabander, F. 2020. Life after Privacy: Reclaiming Democracy in a Surveillance Society (1st ed). Cambridge: Cambridge University Press.

Dal, A. and E. C. Nisbet. 2020. To share or not to share? How emotional judgments drive online political expression in high-risk contexts. Communication Research. Epub ahead of print 19 Aug 2020. 
Daryabar, F., A. Dehghantanha, B. Eterovic-Soric et al. 2016. Forensic investigation of OneDrive, Box, GoogleDrive and Dropbox applications on Android and iOS devices. Australian Journal of Forensic Sciences 48 (6):615-642.

De Wolf, R. 2020. Contextualizing how teens manage personal and interpersonal privacy on social media. New Media \& Society 22 (6):1058-1075.

Dienlin, T. and M. J. Metzger. 2016. An extended privacy calculus model for SNSsAnalyzing self-disclosure and selfwithdrawal in a U.S. representative sample. Journal of Computer-Mediated Communication 21 (5):368-383.

Epstein, D. and K. Quinn. 2020. Markers of online privacy marginalization: Empirical examination of socioeconomic disparities in social media privacy attitudes, literacy, and behavior. Social Media + Society 6 (2):1-13.

Epstein, D., M. C. Roth, and E. P. S. Baumer. 2014. It's the definition, stupid! Framing of online privacy in the Internet Governance Forum debates. Journal of Information Policy 4:144-172.

Esser, F. and T. Hanitzsch. 2013. On the why and how of comparative inquiry in communication studies. In: The Handbook of Comparative Communication Research, eds. F. Esser F and T. Hanitzsch, 3-22. London: Routledge.

Etzioni, A. 1999. The Limits of Privacy. New York: Basic Books.

European Commission. 2011. Special Eurobarometer 359: Attitudes on Data Protection and Electronic Identity in the European Union. http://ec.europa.eu/public_opinion/archives/ ebs/ebs_359_en.pdf

European Commission. 2019. Special Eurobarometer 487a: The General Data Protection Regulation. https://ec.europa.eu/commfrontoffice/public opinion/index.cfm/ResultDoc/download/Do cumentKy/86886

Evans, S. K., K. E. Pearce, J. Vitak J et al. 2016. Explicating affordances: A conceptual framework for understanding affordances in communication research. Journal of
Computer-Mediated Communication 22 (1):35-52.

Fullenwieder, L. and A. Molnar. 2018. Settler governance and privacy: Canada's Indian Residential School Settlement Agreement and the mediation of state-based violence. International Journal of Communication 12:1332-1349.

Goffman, E. 1974. Frame Analysis: An Essay on the Organization of Experience. Cambridge; MA: Harvard University Press.

Henrich, J., S. J. Heine SJ and A. Norenzayan A. 2010. Most people are not WEIRD. Nature 466 (7302):29-29.

Hofstede, G. 1984. Cultural dimensions in management and planning. Asia Pacific Journal of Management 1 (2):81-99.

Jahoda G (2012) Critical reflections on some recent definitions of "culture." Culture \& Psychology 18(3): 289-303.

Kezer, M., B. Sevi, Z. Cemalcilar Z, and L. Baruh. 2016. Age differences in privacy attitudes, literacy and privacy management on Facebook. Cyberpsychology 10 (1).

Koops, B. J., B. Newell, T. Timan et al. 2017. A typology of privacy. University of Pennsylvania Journal of International Law 38 (2):483-575.

Kroeber, A. L. and C. Kluckhohn. 1952. Culture: A critical review of concepts and definitions. Cambridge, MA: Harvard University Press.

Lee, Y. H. and C. W. Yuan .2020- The privacy calculus of "friending" across multiple social media platforms. Social Media + Society 6 (2):1-10.

Li, Y., E.H. R. Rho and A. Kobsa. 2020. Cultural differences in the effects of contextual factors and privacy concerns on users' privacy decision on social networking sites. Behaviour \& Information Technology. Epub ahead of print 15 Oct 2020.

Li, H., L. Yu and W. He. 2019. The impact of GDPR on global technology development. Journal of Global Information Technology Management 22 (1):1-6.

Litt, E. 2012. Knock, knock. Who's there? The imagined audience. Journal of Broadcasting \& Electronic Media 56 (3):330-345.

Livingstone, S. 2003. On the challenges of crossnational comparative media research. 
European Journal of Communication 18 (4):477-500.

Livingstone, S. 2013. Challenges to comparative research in a globalizing media landscape. In: The Handbook of Comparative Communication Research, eds. F. Esser F and T. Hanitzsch, 414-429. London: Routledge.

Luhmann, N. 1995. Social Systems. Palo Alto, CA: Stanford University Press.

Lutz, C. 2016. A social milieu approach to the online participation divides in Germany. Social Media + Society 2 (1):1-14.

Lutz, C., C. P. Hoffmann and G. Ranzini. 2020. Data capitalism and the user: An exploration of privacy cynicism in Germany. New Media \& Society 22 (7):1168-1187.

Lutz, C., M. Schöttler and C. P. Hoffmann. 2019. The privacy implications of social robots: Scoping review and expert interviews. Mobile Media \& Communication 7 (3):412434.

Lutz, C. and P. Strathoff. 2014. Privacy concerns and online behavior-not so paradoxical after all? Viewing the privacy paradox through different theoretical lenses. In Multinationale Unternehmen und Institutionen im WandelHerausforderungen für Wirtschaft, Recht und Gesellschaft, eds. S. Brändli, A. Tamo and R. Schister, 81-102. Bern: Stämpfli.

Lyon, D. 2014. Surveillance, Snowden, and big data: Capacities, consequences, critique. Big Data \& Society $1(2): 1-13$.

Marwick, A. E. and d. boyd. 2014. Networked privacy: How teenagers negotiate context in social media. New Media \& Society 16 (7):1051-1067.

Marwick, A. E. and d. boyd. 2018. Understanding privacy at the margins-Introduction. International Journal of Communication 12:1157-1165.

Marwick, A. E. and E. Hargittai. 2019. Nothing to hide, nothing to lose? Incentives and disincentives to sharing information with institutions online. Information, Communication \& Society 22 (12):16971713.

Masur, P. K. 2018. Situational Privacy and Self-
Disclosure: Communication Processes in Online Environments. Cham: Springer.

Masur, P. K. 2020. How online privacy literacy supports self-data protection and selfdetermination in the age of information. Media and Communication 8 (2):258-269.

Matassi, M. and P. Boczkowski. 2021. An agenda for comparative social media studies: The value of understanding practices from crossnational, cross-media, and cross-platform perspectives. International Journal of Communication 15:207-228.

Maurice, M. 1989. Méthode comparative et analyse sociétale. Les implications théoriques des comparaisons internationales. Sociologie $d u$ travail 31 (2):175-191.

McLeod, J. M. and N. J. Lee. 2012. Comparative research designs: Toward a multilevel approach. In: The Handbook of Comparative Communication Research, eds. F. Esser F and T. Hanitzsch, 430-444. London: Routledge.

Medzini, R. 2021. Credibility in enhanced selfregulation: The case of the European data protection regime. Policy \& Internet. Epub ahead of print 8 Apr 2021.

Melander-Wikman, A., Y. Fältholm and G. Gard. 2008. Safety vs. privacy: Elderly persons' experiences of a mobile safety alarm. Health \& Social Care in the Community 16 (4):337346.

Miltgen, C. L. and D. Peyrat-Guillard. 2014. Cultural and generational influences on privacy concerns: A qualitative study in seven European countries. European Journal of Information Systems 23 (2):103-125.

Murphy, R. F. 1984. Social distance and the veil. In Philosophical Dimensions of Privacy (1st ed), ed. F. D. Schoeman FD (ed), 34-55. Cambridge: Cambridge University Press.

Nassehi, A. 2019. Muster: Theorie der digitalen Gesellschaft. Munich: CH Beck.

Nissenbaum, H. F. 2010. Privacy in context: Technology, policy, and the integrity of social life. Palo Alto, CA: Stanford Law Books.

Omarzu, J. 2000. A disclosure decision model: Determining how and when individuals will self-disclose. Personality and Social Psychology Review 4 (2):174-185. 
Papacharissi, Z. 2009. The virtual geographies of social networks: A comparative analysis of Facebook, LinkedIn and ASmallWorld. New Media \& Society 11 (1-2):199-220.

Papacharissi, Z. 2010. Privacy as a luxury commodity. First Monday 15 (8).

Park, Y. J. 2008. Privacy regime, culture and user practices in the cyber-marketplace. Info 10 (2):57-74.

Park, Y. J. 2013. Digital literacy and privacy behavior online. Communication Research 40 (2):215-236.

Pearce, K. E., J. Vitak and K. Barta. 2018. Socially mediated visibility: Friendship and dissent in authoritarian Azerbaijan. International Journal of Communication 12:1310-1331.

Peter, J. and P. M. Valkenburg. 2011. Adolescents' online privacy: Toward a developmental perspective. In Privacy Online. Perspectives on Privacy and Self-Disclosure in the Social $W e b$, eds. S. Trepte $\mathrm{S}$ and L. Reinecke L, 221-234. Berlin: Springer.

Petronio, S. S. 2002. Boundaries of privacy: Dialectics of disclosure. Albany, NY: State University of New York Press.

Phipps, A. G. 2001. Empirical applications of structuration theory. Geografiska Annaler. Series B, Human Geography 83 (4):189204.

Quinn, K., D. Epstein and B. Moon. 2019. We care about different things: Non-elite conceptualizations of social media privacy. Social Media + Society 5 (3):1-14.

Raynes-Goldie, K. 2010. Aliases, creeping, and wall cleaning: Understanding privacy in the age of Facebook. First Monday 15 (1).

Rennie, E., T. Yunkaporta and I. Holcombe-James. 2018. Privacy versus relatedness: Managing device use in Australia's remote Aboriginal communities. International Journal of Communication 12:1291-1309.

Rössler, B. 2005. The Value of Privacy. Cambridge: Polity.

Sanovich, S., D. Stuka and J. A. Tucker. 2018. Turning the virtual tables: Government strategies for addressing online opposition with an application to Russia. Comparative Politics 50 (3):435-454.

Selby, J. 2017. Data localization laws: Trade barriers or legitimate responses to cybersecurity risks, or both? International Journal of Law and Information Technology 25 (3):213232.

Solove, D. 2008. Understanding Privacy. Cambridge, MA: Harvard University Press.

Stockmann D (2020) Media or corporations? Social media governance between public and commercial rationales. In Advances in Corporate Governance: Comparative Perspectives, eds. Anheier HK and Baums T, 249-268. Oxford: Oxford University Press.

Sundar, S. S., H. Kang, M. Wu et al. 2013. Unlocking the privacy paradox: Do cognitive heuristics hold the key? In CHI '13 Extended Abstracts on Human Factors in Computing Systems, ed. W. E. Mackay, 811-816. WE New York: ACM.

Tamò-Larrieux, A. 2018. Designing for Privacy and its Legal Framework. Cham: Springer.

Taras, V., P. Steel and B. L. Kirkman. 2016. Does country equate with culture? Beyond geography in the search for cultural boundaries. Management International Review 56 (4):455-487.

Taylor, R. D. 2020. "Data localization": The internet in the balance. Telecommunications Policy 44 (8): 102003.

Taylor L, Floridi L and van der Sloot B (eds) (2017) Group Privacy: New Challenges of Data Technologies. New York: Springer International.

Trepte S, and Masur PK (2016) Cultural differences in social media use, privacy, and selfdisclosure: Research report on a multicultural study. Report, University of Hohenheim, Germany, 25 May.

Trepte, S., L. Reinecke, E. B. Ellison et al. 2017. A cross-cultural perspective on the privacy calculus. Social Media + Society 3 (1):1-13.

Turner, J. H. 2005. A new approach for theoretically integrating micro and macro analysis. In The Sage Handbook of Sociology, eds. C. Calhoun, C. Rojec and B. S. Turner BS, 405-422. London: Sage.

van de Vijver, F. J. R. and K. Leung. 1997. Methods and Data Analysis for Cross-Cultural Research. London: Sage.

Vitak, J. 2012. The impact of context collapse and privacy on social network site disclosures. 
Journal of Broadcasting \& Electronic Media 56 (4):451-470.

Warren, S. D. and L. D. Brandeis. 1890. The right to privacy. Harvard Law Review 4 (5):193220.

Westin AF (1967) Privacy and Freedom. New York: Atheneum.

Wu, P. F., J. Vitak and M. T. Zimmer. 2020. A contextual approach to information privacy research. Journal of the Association for Information Science and Technology 71 (4):485-490.

Zuboff, S. 2019. The Age of Surveillance Capitalism: The Fight for a Human Future at the New Frontier of Power. New York: Profile Books. 